TITLE:

\title{
Effect of fingolimod on oligodendrocyte maturation under prolonged cerebral hypoperfusion(Abstract_要旨 )
}

\author{
AUTHOR(S): \\ Yasuda, Ken
}

\section{CITATION:}

Yasuda, Ken. Effect of fingolimod on oligodendrocyte maturation under prolonged cerebral hypoperfusion. 京都大学, 2020, 博士(医学)

ISSUE DATE:

2020-03-23

URL:

https://doi.org/10.14989/doctor.k22336

RIGHT:

DOI: https://doi.org/10.1016/j.brainres.2019.06.013 
